\title{
Working memory and target-related distractor effects on visual search
}

\author{
Alex Bahrami Balani \\ University of Birmingham, Birmingham, England \\ David Soto \\ Imperial College London, London, England \\ AND \\ GLYN W. HuMPHREYS \\ University of Birmingham, Birmingham, England
}

\begin{abstract}
We examined two forms of top-down effects on visual selection: (1) information held in working memory (WM) and (2) the semantic relations between targets and distractors. We found that items held in WM affected search for a different target. This WM-based interference effect generalized across different exemplars, even though participants could remember the specific exemplar on the trial. This argues against a memory top-up account of performance. In addition, there was interference from distractors that were not held in WM but were semantically related to the target. The effects of WM capture and the effects of capture by a distractor related to the target combined additively. The data suggest that task-irrelevant information in WM and task-relevant templates for targets compete separately for selection. The implications for understanding top-down processes in search are discussed.
\end{abstract}

Real-world scenes typically provide the visual system with more information than can be acted on at any given moment. As a consequence, the brain needs to select stimuli that are relevant for current behavioral goals and to filter out irrelevant information. Current theories of visual selection assume that processing is biased toward behaviorally relevant signals on the basis of topdown knowledge held in working memory (WM). For example, according to the biased competition account of selection (Duncan, Humphreys, \& Ward, 1997), topdown knowledge gives a competitive gain to stimuli that match with the information held in WM, allowing these stimuli to be selected over other (behaviorally irrelevant) items. Neurophysiological evidence for this was provided by Chelazzi, Miller, Duncan, and Desimone (1993). They had monkeys hold an item in memory and then use that memory to help them select which of two items they subsequently had to make a saccade to. Chelazzi et al. showed that cells in the inferotemporal cortex responding to the cue maintained their firing during an interval prior to the search display, providing a WM representation for the target. Subsequently, when the search display appeared, the cells were biased in their activity, so that stimuli matching the memory representation had enhanced activity, whereas cells responding to the distractor stimulus were suppressed. Consistent with these data, models of visual search and attention suggest that a template for the search target is maintained in visual WM, which biases perceptual mechanisms to process items that contain target features (Bundesen, 1990; Duncan \& Humphreys, 1989).

Psychological evidence for top-down effects in visual selection has come from studies such as those reported by Hodsoll and Humphreys (2001, 2005). These investigators examined search for targets at the end of a feature continuum versus targets at the midpoint (e.g., large targets among medium and small distractors vs. medium targets among large and small distractors). Search for the end-feature target was efficient ( $<10 \mathrm{msec} /$ item $)$, but only when the participants had foreknowledge of the target on each trial. They suggested that efficient search for the target at the end of a feature continuum occurred only when participants could hold a template for the item in WM.

Recent evidence suggests further that information held in WM can influence visual selection not only when it is relevant, but also when it is irrelevant for the task (e.g., Downing, 2000; Huang \& Pashler, 2007; Olivers, Meijer, \& Theeuwes, 2006; Soto \& Humphreys, 2006, 2007; Soto, Humphreys, \& Heinke, 2006a, 2006b). In a typical experiment, participants have to hold an item in memory and then perform a visual search task for a different target. The item held in memory can sometimes reappear as an irrelevant item in the search display at the location of either

A. B. Balani, a.b.balani@bham.ac.uk 
a target (on valid trials) or a distractor (on invalid trials). Search for the target is affected by the validity of the WM stimulus when it reappears in the search display. Reaction times (RTs) to the target are shorter when the WM cue is valid, and RTs are longer when the cue is invalid, relative to neutral trials where the WM stimulus does not reappear in the search display (see Soto, Heinke, Humphreys, \& Blanco, 2005, for one example). This effect of the cue can occur even when it never falls at the target's location (i.e., when it is always invalid when it reappears). However, the effect is minimized when participants identify the cue but do not hold it in memory. The last results demonstrate that the effect is not just due to bottom-up priming but that the cue must be held in WM. Also, once a match between the WM stimuli occurs, attention can be drawn automatically to the memory stimulus. ${ }^{1}$

The guidance of visual attention can be affected not only by holding a different item from the target in WM, but also by the semantic relations between items in the search display and the target. Moores, Laiti, and Chelazzi (2003) examined search for a target (e.g., a motorbike), and presented a related item (e.g., a crash helmet) as a distractor. They found that RTs were lengthened by the related distractors (particularly on trials on which the target was absent), and first saccades went more often to the related distractors than to other distractor stimuli (see also Belke, Humphreys, Watson, Meyer, \& Telling, 2008). These data can be accounted for in at least two ways. One is that there is a spread of activation from the template of the target held in WM to templates for related stimuli, with the result that these related stimuli then compete strongly for selection; the second one is that semantic information is rapidly extracted from stimuli that attract attention by matching the template for the target. In the first case, there is competition between templates, whereas, in the second, there is competition between display items for a single (target) template. The competition between templates can be viewed as being functionally equivalent to the competition for selection when a representation of an irrelevant item, as well as the target, is held in WM (Downing, 2000; Moores \& Maxwell, 2008; Olivers, 2009; Soto et al., 2005).

In the present study, we set out to examine the nature and relationship between these two different inadvertent effects of distractors on search: (1) the effects of an irrelevant item in WM and (2) the effects of a semantic distractor. We report six experiments. Experiments 1 and 2 examined the specific effects of re-presenting an item held in WM as a distractor in search. Experiment $1 \mathrm{~A}$ assessed the effects of bottom-up priming, and Experiment 1B examined effects with the same items when the first item was held in WM. Experiment 1B also tested whether the attraction of the WM distractor generalized across different exemplars, under conditions in which a specific representation of this item had to be remembered. Generalization of attention to a different exemplar would suggest an interesting contrast between the specificity of information in WM (which must be exemplar specific to support good memory performance) and the information that attracts attention through the WM representation (which may be less specific than the WM itself). Experiment 2 assessed whether the effect also would generalize between pictorial and verbal representations of the stimuli and whether the effects were thus unrelated to visual similarity between the item in WM and the target. Experiment 3-6 assessed the relations between the effects of re-presenting the WM item as a distractor and the effects of presenting a new distractor that was semantically related to the target. In these experiments, a new distractor could appear in the display that belonged to the same category as the target. Across different experiments, this new distractor could also be related to or unrelated to the item in WM, and it could be present in the search display (on target-absent trials) along with the WM stimulus. Do representations related to the target compete in the same way for selection with the representations of irrelevant items in WM? By pitting effects of task-irrelevant representations in WM against effects due to semantically related distractors, we learn for the first time about how both task-relevant and task-irrelevant representations modulate visual attention.

\section{GENERAL METHOD}

All the experiments were designed, conducted, and analyzed in the E-Prime environment (Psychology Software Tools, 1996-2006), using a Pentium IV PC with a color monitor (Samsung Sync Master 793s with 1,024 $\times$ 768 pixel resolution and a screen refresh rate of $85 \mathrm{~Hz}$ ). The viewing distance was about $60 \mathrm{~cm}$. The experiment room was dimly lit.

Each experiment took the same form. First, a fixation point, which was an asterisk $2 \mathrm{~cm}$ in diameter, appeared in the middle of the screen, followed by a to-be-remembered stimulus presented at the same place as the fixation point. In different experiments, the memory stimulus was either a colored digital image of a commonly recognizable animal, a familiar, commonly recognizable everyday object, or a simple three-letter word shown on a computer screen (each stimulus measured about $4 \times 6 \mathrm{~cm}$ in size) for a duration of $1 \mathrm{sec}$. The search array consisted of two images, centered on the location of fixation point, $10-15 \mathrm{~cm}$ apart and with the center of each picture 5-6 cm from fixation. The search stimuli were drawn at random from five possible objects of the same category. The memory test appeared after the participants responded in the search task (see Figure 1 for an example display).

The search display ${ }^{2}$ always consisted of two stimuli: one to the left and one to the right of the center of the screen. The search target could be an animate object (a picture of a dog in Experiments 1, 3, 5, and 6A; the word dog in Experiments 2 and 4) or an inanimate object (a picture of a cup in Experiment 6B). Likewise, the objects used as WM stimuli could be animate or inanimate. In Experiments 1-4, the WM item was a cat; in Experiment 6B, it was a dog; and in Experiments 5 and 6A, it was a cup. There were different types of trial, defined by the presence of the memory object in the search task. On target-present trials, the target (e.g., a dog) appeared along with (1) the same object presented as the memory stimulus, (2) a different exemplar of the memory stimulus, or (3) a different stimulus (not the item held in memory). On target-absent trials, two stimuli other 


\section{Time}

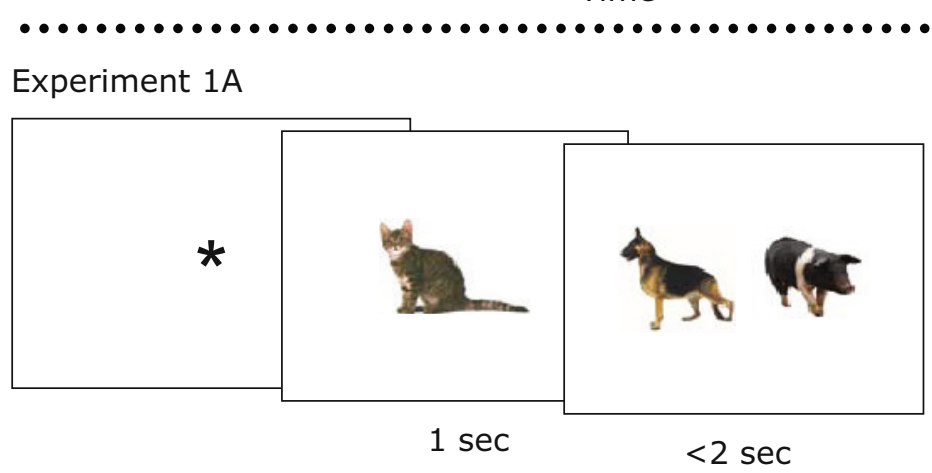

Experiment $1 \mathrm{~B}$

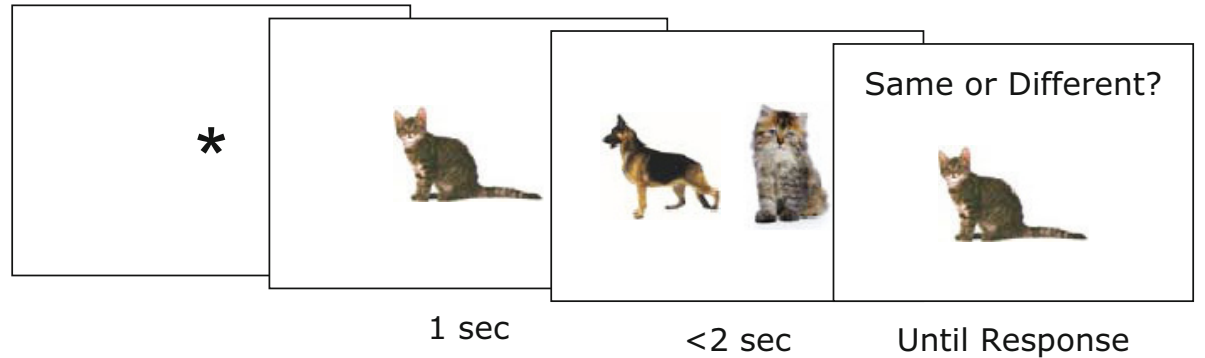

Figure 1. Schematic presentation of the sequence of stimuli in Experiments 1A and 1B. The upper figure displays a neutral trial where the target (the dog) is present in the search display along with another animal (a pig). The lower figure displays an example of an invalid trial where the target (the dog) is present and another exemplar of the working memory stimulus (a different cat) appears in the search display. A target-present response is expected. A memory trial follows that shows a same memory probe.

than the target stimulus appeared on the screen: (4) on WM repeat trials, the memory item reappeared in the display along with another distractor, and (5) on neutral trials, neither of the items matched the memory stimulus. In some experiments, there could be two different types of neutral trials: neutral same (distractors from the same category as the target) and neutral different (distractors from a different category than the target). The order of the trials varied randomly in each experiment. The different types of trials in each experiment appeared equally often.

Search responses were registered by participants' pressing a key on a computer keyboard. Memory probe responses were not speeded and were reported verbally to the experimenter, who registered the response using the computer mouse. In all the experiments, each trial started with a 1-sec presentation of an image of an animate or inanimate cue object. After the cue disappeared, there was an interval of $175 \mathrm{msec}$, followed by the search display. The participants reported the presence or absence of the predesignated target by a keypress, and their search was terminated by this response. If no response was given, the exposure time was restricted to $2 \mathrm{sec}$. The participants responded by pressing "C" when the target stimulus appeared on the left side of the center of the screen, " $\mathrm{M}$ " when the target stimulus appeared on the right side of the screen, or the space bar when no target stimulus was present.

In addition to the search trials, all the experiments (except for Experiment 1A) contained memory probe trials.
On memory probe trials, an image appeared at the center of the screen on one third of the trials after completion of the search task, and the participant was asked to decide whether the image was the same as or different than the memory stimulus. On different trials, a different exemplar of the memory object was used, to ensure that the participants held a visual description of the stimulus in WM. There was a $50 \%$ probability that the memory stimulus would appear on the probe trials. Behavioral data (response times [RTs], accuracy on search task, and accuracy on the memory task) were collected. Detection of outliers was done for each experimental condition and interindividually. All outlier RTs $( \pm 2 S D$ s) were removed from the statistical analysis. Only RTs from accurate trials were included in the RT analyses.

All the participants were university students who voluntarily signed up to participate and were paid a small payment $(£ 6 / \mathrm{h})$ or received course credit if they so wished.

\section{EXPERIMENT 1 Exemplar Effects With Pictures}

Experiment 1 consisted of two sections. In Experiment $1 \mathrm{~A}$, we obtained baseline data on the effects of bottom-up priming from an initial cue on search, to compare with results when bottom-up priming was combined with top-down priming (when the cue was held in WM; in Experiment 1B). In both subexperiments, participants 
had to search for a predefined target in a two-item display, which was preceded by a 1 -sec presentation of a cue item. In Experiment 1A, the participants needed only to attend to the cue (see Soto et al., 2005), ${ }^{3}$ but in Experiment 1B, they had to hold the cue item in memory. In both experiments, the presentation conditions leading to the search display remained the same.

\section{Experiment 1A \\ Baseline Performance}

Experiment 1A was a baseline condition whose aim was to test the effects of bottom-up priming from the cue on subsequent search. The conditions were matched to those in Experiment 1B, but the participants were instructed simply to attend to the initial stimulus. In prior studies using simple colored, geometrical shapes, bottomup priming alone typically produced only a small effect on subsequent selection (e.g., Soto et al., 2005). However, the same might not occur here, when known identifiable shapes were used; this was assessed.

\section{Method}

Twenty-one young students participated (median age, 22 years). There were 14 female and 7 male participants, all with normal or corrected-to-normal vision. The method was the same as that described in the General Method section. When the target was absent, there were three conditions: (1) a neutral condition (two exemplars of a new stimulus from the same superordinate category as the target [two different pigs]); (2) the WM-repeat/same-exemplar condition (the cued stimulus [same cat as in the WM cue] was re-presented, along with a new distractor from the same category as the target [e.g., a pig]); and (3) the WM-repeat/different-exemplar condition (a different exemplar of the cued stimulus [another cat] appeared with a new distractor from the target category [a pig]).

When present, the target (dog) was paired with (1) a repeat of the cue stimulus (the same cat; cue-repeat/same); (2) a different exem- plar of the WM cue (a different cat; cue-repeat/different), or (3) a neutral item from the same category ( pig).

There were five different exemplars of each animal, which were drawn at random and varied across trials. In the search display, the target was present on half the trials, equally often on the left or right of fixation. There were 15 trials per condition, making a total of 90 per block. The conditions were presented randomly. The procedure is illustrated in Figure 1.

\section{Results}

The RT data for this and the remaining experiments were prepared by discarding all errors and removing RTs that were $\pm 2 S D$ s from the individual's mean in that condition. This led to a loss of $2.4 \%$ of the data. This held also for the later experiments.

The RT data were analyzed using a two-factor repeated measures ANOVA on correct RTs, with the factors being target presence (present/absent) and distractor type (neutral, WM-repeat/same, and WM-repeat/different). There was a main effect of target presence $[F(1,20)=7.17, p<$ $\left..014, \eta^{2}=.264\right]$, but there was no effect of distractor type $\left[F(2,40)=1.9, p>.16, \eta^{2}=.007\right]$. There was also an interaction between target and distractor type $[F(2,40)=$ $\left.3.35, p<.04, \eta^{2}=.143\right]$. On target-absent trials, there was a significant effect of distractor type $[F(2,40)=4.49$, $\left.p<.01, \eta^{2}=.184\right]$. Breaking down this effect showed that there were no significant differences between neutral trials and WM-repeat/same trials $[t(20)=-1.4, p>$ .17], no differences between WM-repeat/same and WMrepeat/different trials $[t(20)=1.8, p=.09]$, but there was a significant difference between WM-repeat/different and neutral trials $[t(20)=-2.63, p<.01]$. There was no effect of the WM cue on target-present trials. Figure 2 shows the RT data (from Experiment 1 only), and Table 1 shows the accuracy data.

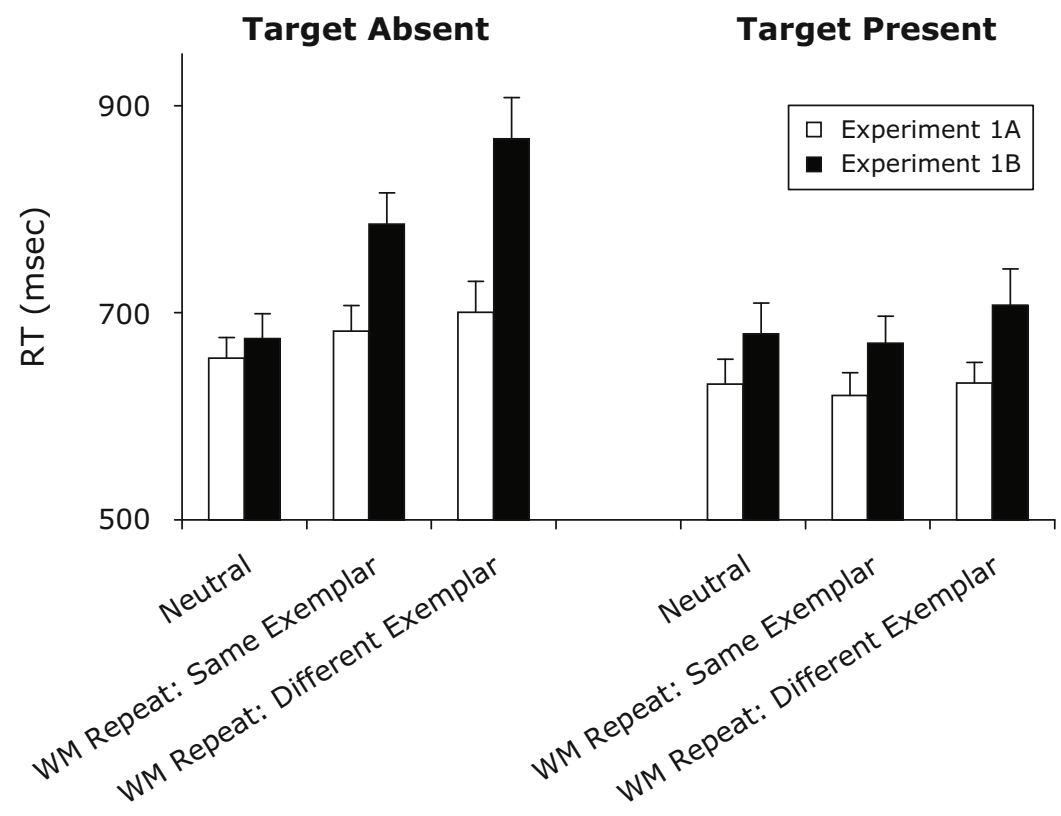

Figure 2. Mean correct response times (RTs, in milliseconds) on target-present and target-absent trials in Experiments $1 \mathrm{~A}$ and 1B. Error bars indicate standard errors. WM, working memory. 
Table 1

Percentage of Errors in Experiments 1-6

\begin{tabular}{|c|c|c|c|c|c|c|c|c|}
\hline \multirow[b]{3}{*}{ Experiment } & \multicolumn{4}{|c|}{ Target-Absent Trials } & \multicolumn{4}{|c|}{ Target-Present Trials } \\
\hline & \multicolumn{2}{|c|}{ Neutral } & \multicolumn{2}{|c|}{ WM Repeat } & \multicolumn{2}{|c|}{ Neutral } & \multicolumn{2}{|c|}{ WM Repeat } \\
\hline & Same & Different & Same & Different & Same & Different & Same & Different \\
\hline $1 \mathrm{~A}$ & 1.0 & N/A & 3.0 & 1.0 & 1.0 & $\mathrm{~N} / \mathrm{A}$ & 2.0 & 1.0 \\
\hline $1 \mathrm{~B}$ & 2.2 & N/A & 3.2 & 3.1 & 1.0 & $\mathrm{~N} / \mathrm{A}$ & 2.8 & 3.0 \\
\hline 2 & 0.25 & $\mathrm{~N} / \mathrm{A}$ & 1.8 & $\mathrm{~N} / \mathrm{A}$ & 0.5 & $\mathrm{~N} / \mathrm{A}$ & 0.5 & $\mathrm{~N} / \mathrm{A}$ \\
\hline 3 & 1.0 & 3.2 & 1.5 & $\mathrm{~N} / \mathrm{A}$ & 0.5 & 0.0 & 3.2 & 3.1 \\
\hline 4 & 1.7 & 1.8 & 0.8 & $\mathrm{~N} / \mathrm{A}$ & 0.5 & 0.0 & 0.0 & 1.2 \\
\hline \multirow[t]{3}{*}{5} & 1.5 & 1.0 & 0.7 & 1.4 & 1.3 & 0.9 & 3.8 & 1.3 \\
\hline & \multicolumn{2}{|c|}{ Two } & \multicolumn{2}{|c|}{ WM } & \multicolumn{2}{|c|}{ Target } & $\begin{array}{l}\text { Target } \\
\text { and }\end{array}$ & \\
\hline & Related & Unrelated & Related & Unrelated & Related & Unrelated & WM & \\
\hline $6 \mathrm{~A}$ & 1.3 & 2.9 & 9.1 & 4.0 & 8.0 & 2.1 & 2.2 & \\
\hline $6 \mathrm{~B}$ & 2.0 & 1.4 & 2.2 & 6.5 & 7.1 & 1.1 & 1.9 & \\
\hline
\end{tabular}

Note-WM, working memory.

Percentage of erroneous trials varied between $1 \%$ and $3 \%$ (see Table 1). There was no evidence of a speedaccuracy trade-off, and the data were not analyzed further.

\section{Discussion}

In this baseline study, target-present trials were faster than target-absent trials, as is commonly found in visual search. There was no effect of participants' attending to the cue stimulus on target-present trials, but effects emerged on target-absent trials (the mean difference between trials with displays that repeated the WM item and when it was absent was $37 \mathrm{msec}$ ). In prior studies using simple geometric shapes, mere identification has proved insufficient to have an effect on later attentional guidance when the original stimulus reappears in the search display (e.g., see Soto et al., 2005). With the familiar stimuli presented here, there may be some effect of mere presentation of the cue, through bottom-up priming of the representation of the stimulus. In Experiment 1B, we examined whether stronger interference from the cue would emerge when the participants had to keep the stimulus in WM for later retrieval.

\section{Experiment 1B Effect of WM on Search}

\section{Method}

There were 28 undergraduate students (median age, 19 years) who participated. There were 5 male and 23 female participants, all with normal or corrected-to-normal vision. The method here mimicked that of Experiment $1 \mathrm{~A}$, except that the cue had to be memorized (see Soto et al., 2005). In the memory task, participants responded same when the same exemplar (of the cue item) was re-presented and different when a new exemplar appeared. There were 15 trials per condition, making a total of 90 per block. The conditions were presented randomly. (The lower part of Figure 1 illustrates the procedure.)

\section{Results}

The RT data are presented in Figure 2. The data were analyzed using a $2 \times 3$ factors repeated measures ANOVA, with the factors being target presence (present/absent) and distractor type (WM-repeat/same, WM-repeat/different, neutral). There were main effects of target presence $\left[F(1,27)=42.4, p<.0001, \eta^{2}=.611\right]$ and distractor type $\left[F(2,54)=25.63, p<.001, \eta^{2}=.487\right]$. There was also an interaction between target presence and distractor type $\left[F(2,54)=38.01, p<.0001, \eta^{2}=.585\right]$. The interaction was broken down by analyzing the data separately for present and absent trials. On absent trials, there was an effect of distractor type $[F(2,54)=47.07, p<.0001$, $\left.\eta^{2}=.640\right]$. RTs were reliably larger for search displays when the WM stimulus was repeated as a distractor than for displays in the neutral condition $[t(27)=-8.47, p<$ .0001 , and $t(27)=-6.53, p<.0001$, for trials with different and same exemplars of the WM stimulus, respectively]. A comparison of the same- and different-exemplar versions of the WM repeat conditions also revealed a significant difference $[t(27)=4.24, p<.001]$. RTs were longer on different- than on same-exemplar trials. On target-present trials, a significant effect of distractor type was also found $\left[F(2,54)=3.66, p<.04, \eta^{2}=.11\right]$. A breakdown of the data showed that RTs were longer in the different-exemplar condition than in the same-exemplar condition $[t(27)=2.5, p<.01]$, and there was a trend for RTs in the different-exemplar condition to be longer than those in the neutral condition $[t(27)=1.6, p<.06]$. There were no significant differences between the WM-repeat/ same and neutral conditions $[t(27)=1.1, p<.09]$.

Accuracy for the search task was generally high (mean, $98 \%$ ), and errors did not exceed $3.2 \%$ in any condition. The data are shown in Table 1 . There was no evidence of a speed-accuracy trade-off, and no further analysis of the error rates was conducted. The accuracy rate on memory probe task was also high (89\%), which indicates good encoding of the WM stimulus. Performance on the memory task did not vary as a function of either cue validity or target presence (all $F_{\mathrm{S}}<1$ ).

\section{Experiment 1A Versus Experiment 1B}

To assess whether the WM effects (in Experiment 1B) differed reliably from the bottom-up priming effects (in Experiment 1A), the data from the two subexperiments were compared in two separate ANOVAs on correct RTs 
from target-present and target-absent trials. The factors were distractor type (neutral, WM-repeat/same, and WM-repeat/different) and experiment (Experiments 1A and 1B). On target-absent trials, there was a significant effect of distractor type $[F(2,94)=39.96, p<.0001$, $\left.\eta^{2}=.459\right]$ and an overall difference between the experiments $\left[F(1,47)=3.35, p=.047, \eta^{2}=.066\right]$. There was also an interaction between distractor type and experiment $\left[F(2,94)=13.64, p<.0001, \eta^{2}=.225\right]$. The cue had a stronger effect when it reappeared as a distractor in Experiment 1B (WM condition), as compared with Experiment $1 \mathrm{~A}$ (priming condition). On target-present trials, there was no difference between Experiments $1 \mathrm{~A}$ and 1B $(F<1)$, no significant effect of distractor type $[F(2,94)=$ $1.9, p>.16]$, and no reliable interaction between distractor type and experiment $[F(2,94)=1.77, p>.17]$. Figure 2 depicts the data.

\section{Discussion}

In Experiment 1B, RTs varied as a function of whether the memory cue reappeared in the search display; RTs were longer when either the memory stimulus or a different exemplar of this stimulus reappeared, relative to the neutral condition where a new item from the same superordinate category reappeared.

Interestingly, the effect of re-presenting the $\mathrm{WM}$ cue was at least as strong when a different exemplar of that item reappeared as when the memory item itself reappeared. ${ }^{4}$ This was despite the fact that the memory task required the participants to hold in memory a representation of the exact exemplar and this task was performed at a high level. This suggests that there is some differentiation between the specificity of the memory representation being held and the level of representation that determines whether a match between the memory item and the search display influences attention. Even though a detailed memory representation appeared to be held, attention was directed on the basis of a coarser or more abstract representation that matched across different exemplars. Recently, Soto and Humphreys (2007) found an effect of a WM stimulus on search even when the stimuli were presented in different modalities (in their case, the WM stimulus was verbal, and the search stimuli were visual shapes). They suggested that a semantic representation of the WM stimulus might be matched rapidly against semantic information derived from the search display and that this semantic-level match could direct attention to the memory item when it reappears in the search display. In the present case, the WM stimulus cannot be represented only at an abstract level, however, given that the memory match task had to be made at the level of specific exemplar. Nevertheless, semantic information may be accessed by the stimulus in WM, and any match between this stimulus and information about the basic level semantic attributes of the stimuli in the display may determine search.

The effects of a distractor's matching the WM item, or being related to the target, were more pronounced on target-absent than on target-present trials. This matches previous data (e.g., Belke et al., 2008; Houtkamp \& Roelfsema, 2006; Moores et al., 2003). We assume that the tar- get is assigned the highest attentional weight for search, with the next higher weight given to other items in WM (cf. Duncan \& Hupmhreys, 1989). This means that a distractor related to the item in WM, or to the target, will only exert a small effect on performance when it has to compete for selection with the target (on target-present trials). On target-absent trials, however, the critical distractor will tend to be selected, rather than any unrelated distractor. This will lengthen RTs either because (1) selecting the critical distractor biases a present response or because (2) there is a disengagement cost of selecting a distractor that matches information held in WM. In either case, RTs will be delayed.

One other aspect of the results from Experiment 1 should also be noted. This concerns the assumption of whether participants might deliberately attend to the memory item in the search display in order to refresh their memory of the cue (see Olivers et al., 2006). This seems an unlikely explanation of the present data. It was observed that a different exemplar of the memory item was at least as potent a cue for attention as a repeat of the memory stimulus itself. However, the different exemplar ought to disrupt memory for the specific exemplar (and a different exemplar would have to be rejected when it reappeared as a memory item), and thus, it should not be attended deliberately. On top of this, memory performance did not vary as a function of whether the same item or a different exemplar of the memory cue appeared in the search display. This contradicts the idea that the search display was deliberately used to refresh the WM representation.

\section{EXPERIMENT 2 Effects on Word Search}

Experiment 1B added a WM component to the initial representation of a cue and demonstrated effects of WM on target selection. In Experiment 2, we extended this result by removing effects of the initial visual cue. The procedure was the same as that in Experiment 1, except that the pictures in the search display were replaced by printed words. The participants still had to respond to the presence of the target $d o g$, but in this case, performance might be determined by an orthographic and/or phonological representation of the search target and any bottom-up priming effects should be minimal. Would performance still be affected by a picture held in WM?

\section{Method}

The first display was presented as a memory object, and it always showed a picture of a cat. The second display contained two printed words representing the names of two animals (cat, pig, or dog). Figure 3 illustrates the procedure.

There were four different types of display: two with the search target absent, and two with the search target present. On target-absent trials, the word pig either appeared with the word cat (WM repeat) or appeared twice at the same time on the screen on either side of the center of the screen (neutral condition). On target-present trials, the word $d o g$ appeared with either the word cat (WM repeat) or the word pig (neutral). The location of the target was chosen randomly and equally often on the left or right of fixation. Twenty-eight students (median age, 19 years) participated. There were again 5 male and 23 female participants, all with normal or corrected-to-normal 
Time

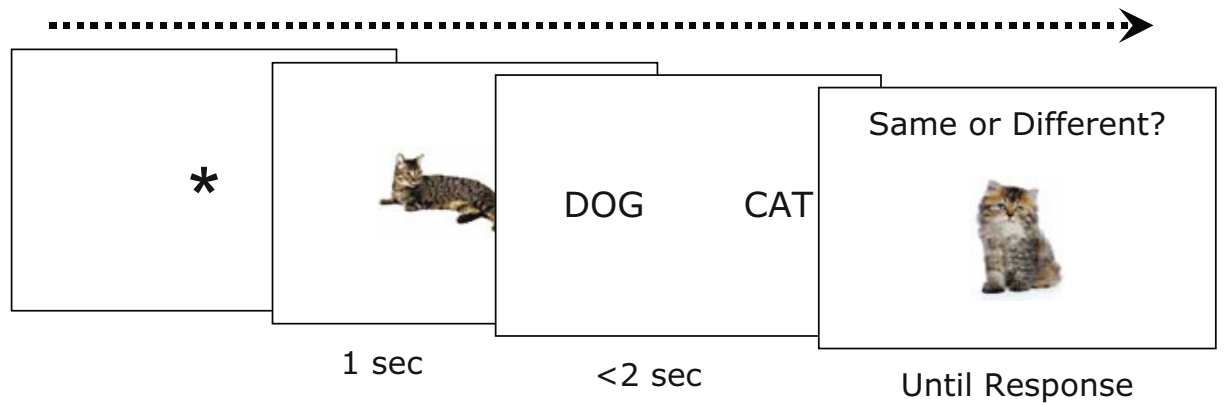

Figure 3. Schematic presentation of a trial in Experiment 2. On this trial, a word defining the working memory stimulus appeared as a distractor in the search display. A present response is expected. A memory probe (showing a different memory response) follows the search display.

vision. There were 15 trials per condition, making a total of 60 . The conditions were presented randomly. All the participants completed all 60 trials.

\section{Results}

The analysis of correct RTs showed main effects of target presence $($ present/absent) $[F(1,27)=44.29, p<.0001$, $\left.\eta^{2}=.621\right]$ and distractor type $[F(1,27)=11.43, p<.002$, $\left.\eta^{2}=.298\right]$. There was also an interaction between target presence and distractor type $[F(1,27)=10.42, p<.003$, $\left.\eta^{2}=.279\right]$. On target-absent trials, RTs were lengthened when the memory item reappeared in the search display as a distractor (on WM repeat trials), as compared with when the distractor was a different item from the same category (neutral trials) $[t(27)=-3.83, p<.001]$. On target-present trials, there was no effect of distractor type $(F<1)$. The data are shown in Figure 4.

The error rates on the search task were low (less than $1.8 \%$ ), and they were not analyzed further (see Table 1). Accuracy on the memory probe task was high (95\%). Performance on the memory task did not vary as a function of whether the memory item reappeared in the search display or whether the target was present $(F<1)$.

\section{Cross-Experimental Comparison Between Experiment 1B and Experiment 2}

To compare the effects of a pictorial item in WM on search for picture and word targets, we compared the data from Experiments $1 \mathrm{~B}$ and 2. For this, we averaged the same- and different-exemplar trials from Experiment 1B. A $2 \times 2 \times 2$ ANOVA showed significant effects of experiment $\left[F(1,27)=33.42, p<.0001, \eta^{2}=.55\right]$, distractor type $\left[F(1,27)=79.4, p<.0001, \eta^{2}=.746\right]$, and target presence $\left[F(1,27)=50.26, p<.0001, \eta^{2}=.650\right]$. There were also interactions between experiment and distractor type $\left[F(1,27)=9.43, p<.005, \eta^{2}=.26\right]$ and a threeway interaction between experiment, target presence, and distractor type $\left[F(1,27)=5.92, p<.02, \eta^{2}=.18\right]$. Taking target-absent trials only, there was a reliable main effect of distractor type $\left[F(1,27)=85.96, p<.0001, \eta^{2}=\right.$ $.45]$ and an interaction of distractor type and experiment $\left[F(1,27)=10.67, p<.003, \eta^{2}=.28\right]$. On target-present trials, there was no effect of distractor type and no interac- tion with the experiment (both $F \mathrm{~s}<1$ ). On absent trials, the effects of distractor type were larger in Experiment 1B (pictorial target; effect size of $152 \mathrm{msec}$ ) than in Experiment 2 (word target; effect size of $57 \mathrm{msec}$ ).

\section{Discussion}

The data demonstrated an effect of the WM stimulus on search through a word display where, at least on absent trials, attention was directed to a distractor matching the WM stimulus, rather than to another distractor. This result is consistent with attention's being directed on the basis of a match between relatively abstract representations of the stimuli, enabling attention to be biased toward a word on the basis of information represented from a picture in WM. As in Experiment 1B, the WM representation itself had to be held at the level of the specific stimulus. Nevertheless, more abstract information activated by that stimulus served to direct search to a (semantically) matching item.

Although there were effects of repeating the WM stimulus in the display here, they were weaker than the effects observed in Experiment 1B. There are at least two reasons why this might be the case. One is that, when the search stimuli are pictures, matches between their visual features and the visual features of the stimulus held in WM may

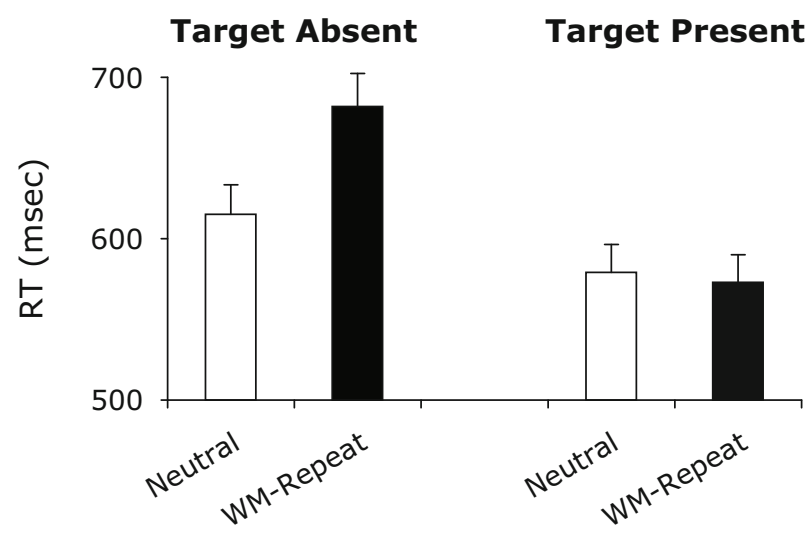

Figure 4. Mean correct response times (RTs, in milliseconds) in Experiment 2, with a picture held in working memory (WM) and words in a search display. Error bars indicate standard errors. 
modulate selection. This might have occurred even though different exemplars were as effective as distractors as the same exemplars, if visual matching was based on features in common across exemplars. The second possibility is that semantic information may be accessed more rapidly from pictures than from words (cf. Potter \& Faulconer, 1975), with words being re-presented orthographically or phonologically, as well as semantically (cf. Coltheart, Rastle, Perry, Langdon, \& Ziegler, 2001). The more rapid semantic access for pictures and the orthographic or phonological, rather than semantic, coding of words would generate stronger effects of semantic matching in Experiment 1B (with picture targets), as compared with Experiment 2 (with word targets).

\section{EXPERIMENT 3 Effects of Semantic Distractors}

The data from Experiment 2 suggest that search can be influenced by matches between relatively abstract (semantic) matches between WM representations and search displays. However, the specificity of the semantic information has not been examined. In Experiments 1 and 2, the re-presentation of the WM stimulus was contrasted with a neutral baseline in which the distractors were stimuli from the same category as both the WM and the target. The differences relative to this baseline have indicated effects of matching modulated by basic-level representations of the stimuli, since in Experiment 1B, the distractor effect was as strong with different exemplars as with same exemplars of the WM stimulus. This does not mean, however, that there are no effects of matching at a semantic level, either to the item in WM or to the target; it is simply that this will not be seen when the neutral distractor also belongs to the same category as the target and the WM item. Many theories of semantic access level have assumed that there is rapid access to superordinate semantic information about stimuli (e.g., Collins \& Quillian, 1969; Riddoch \& Humphreys, 1987). This suggests that it may be possible to demonstrate effects of superordinate matching between the WM stimulus and the search display (e.g., superordinate information is extracted from the search display and is used to guide search). In addition to this, as was noted in the introduction, Moores et al. (2003) have demonstrated disruptive effects on search from presenting a new distractor that was semantically related to the target. This related distractor effect reflects the semantic similarity between items in the search array and the target template held in WM. On the basis of this effect, we would also expect search to be slowed when the novel distractor in the search display belongs to the semantic category of the target. We tested this in Experiment 3 by introducing a second neutral baseline. In the neutral (same-category) condition, the distractor was a new object (not shown as the memory cue), but from the same category as the WM cue and the target (i.e., a related distractor). In the neutral (different-category) condition, the distractor was a new object, but from a category different from that of both the WM stimulus and the target. If RTs are lengthened in the neutral same-category condition, relative to the neutral different-category condition, this would suggest that matches from semantic information derived from the search display level also influence selection.

\section{Method}

As in Experiment 1B, the memory stimuli were different cats, and the targets were different exemplars of dogs. On target-absent trials, four conditions were created: (1) neutral same, in which two stimuli were presented and both were from the same superordinate category as the WM cue and the target (e.g., pig + hen); (2) neutral different, with two new stimuli, both from a superordinate category different from that for the WM cue and the target (e.g., chair + cup); (3) WM-repeat/same, with the same exemplar as the WM cue, along with another distractor from the same superordinate category (e.g., cat + pig); and (4) WM-repeat/different, with the same exemplar as the WM item (same cat) plus a distractor from a different category (e.g., cat + hat). On target-present trials, there were three conditions created by introducing different distractors: target (dog) plus (1) a new distractor from the same superordinate category as both the target and the WM cue (dog + e.g., pig) (the neutral same condition); (2) a new distractor from a different category (dog + e.g., cup) (the neutral different condition); and (3) the same exemplar as the WM cue (dog + the same cat) (WM repeat). There were 12 trials in each condition, generating 84 trials in total. Trials and display types were presented randomly. Twenty-eight participants were tested (23

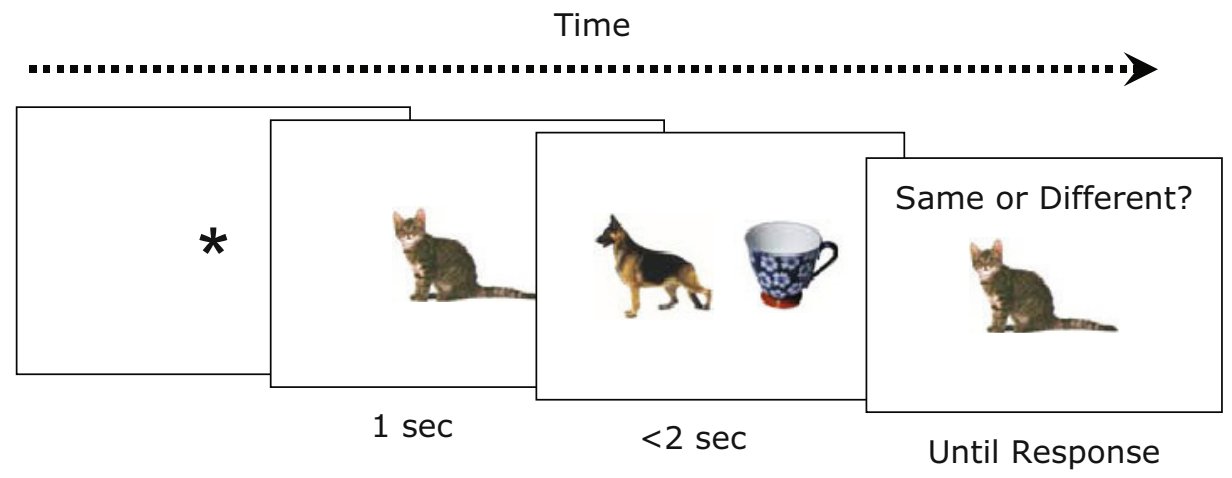

Figure 5. Schematic presentation of a trial in Experiment 3. This shows a neutral target-present trial where the working memory item is not repeated. Here the target (dog) appears along with an inanimate distractor. A present response is expected. A same memory probe is also depicted (showing a same memory response), following the search. 
females, 5 males; median age $=19$ years). An example trial is illustrated in Figure 5.

One difference, relative to the earlier experiments, was that images of inanimate everyday objects (a cup, hat, chair, jug, or saucepan) were used on different category trials as distractors in the search array. The task was the same as that in previous experiments: The participants had to perform a visual search task for a dog while maintaining a specific exemplar of a second stimulus (a cat) in WM.

\section{Results}

Since the conditions could not be balanced across absent and present trials (there were three present conditions, but four conditions on absent trials), separate repeated measures ANOVAs were carried out on correct RTs for each response type. The absent trial data were analyzed using a $2 \times 2$ ANOVA, with the factors being WM presence (present/absent), and distractor category (same/different). There were reliable main effects of WM presence $\left[F(1,27)=33.2, p<.0001, \eta^{2}=.552\right]$ and distractor category $\left[F(1,27)=55.48, p<.0001, \eta^{2}=.673\right]$ and also an interaction between WM presence and distractor category $\left[F(1,27)=9.22, p<.005, \eta^{2}=.255\right]$. The data showed that search was slowed when the WM stimulus was re-presented, and also when the distractor belonged to the same category as the target and the WM stimulus. Figure 6 illustrates the RT data.

The effect of the new related distractor was greater when the WM cue was absent (a difference of $90 \mathrm{msec}$ ), relative to when the WM cue was present (a difference of $30 \mathrm{msec}$ ). Nevertheless, there was a reliable effect of the related distractor on both neutral trials $[t(27)=8.2$, $p<.0001]$ and WM-repeat/same-category trials $[t(27)=$ $-5.76, p<.0001]$.

On target-present trials, there was an effect of trial type $\left[F(2,54)=13.09, p<.0001, \eta^{2}=.320\right]$. Paired-samples $t$ tests showed significant differences between trials in which the WM exemplar reappeared, relative to both when the distractor was a new related item $[t(27)=-3.9, p<$
$.001]$ and when the new distractor was from a different category $[t(27)=-4.48, p<.0001]$. There was no effect, in this case, of whether the new distractor was related or neutral $[t(27)=1.54, p>.13]$.

Error rates on the search task varied between $0 \%$ and $3.2 \%$. There was no indication that a speed-accuracy tradeoff occurred (see Table 1). Accuracy on memory probe task was high (94\%), and it was unaffected by presence or absence or the nature of the distractor (all $F_{\mathrm{S}}<1$ ).

\section{Discussion}

The data from Experiment 3 confirm the interfering effect of the WM cue when it reappeared as a distractor in the search display, with interference observed here on present as well as absent trials. In addition, there was also some evidence for interference from a related distractor that belonged to the same category as the WM cue and the target. On absent trials, RTs were longer to displays in the neutral same-category condition (e.g., when two different animals were presented), as compared with displays in the neutral different-category condition (animals + inanimate objects).

The distractor from the same category as the WM cue and target did not disrupt performance as much as a repeated WM stimulus, but nevertheless, a reliable effect was found. This result suggests that semantic similarity between templates in memory and new search stimuli can influence search, although it is unclear from this result whether semantic similarity to the target or the WM stimulus is critical. As we have noted, Moores et al. (2003) showed that semantic similarity between an expected target and a related distractor affected search. However, in our case, there may also be effects of the semantic association between the new distractor and the item held in WM. In addition to this, our conclusions about effects of semantic similarity need to remain tentative, because it is possible that visual similarity between the same-

Target Absent

Target Present

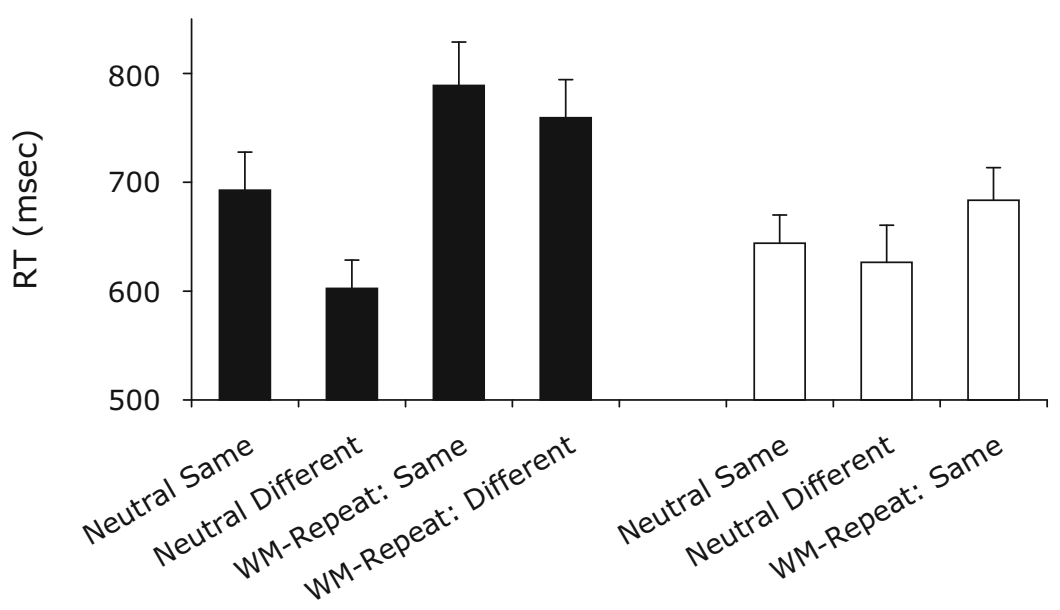

Figure 6. Mean correct response times (RTs, in milliseconds) in Experiment 3, when new related distractors or new neutral distractors were used. Both the memory and search stimuli were pictures. Error bars indicate standard errors. WM, working memory. 
category distractor and the WM stimulus and/or the target determined performance, since pictures of items from the same category often contained similar features (e.g., with animals as targets and as WM exemplars). These different accounts of new related distractor were examined in Experiment 4.

One other point to note is that, on target-absent trials, there was an interaction between the presence of WM distractor and the novel semantic distractor. When the WM item reappeared in the search display, there was a decreased effect of having the new distractor from the same category as the target and the WM item. Such a decreased effect can be expected if the WM tends to dominate any race to capture attention (relative to when a new, related distractor appears). Thus, when both types of distractor cue are present, any effect of the new related distractor is small, since the repeated WM cue usually wins the race for selection. Nevertheless, the fact that there was some increment when the WM item was accompanied by a new related distractor suggests that there may have been some competition between these items for selection. This would slow selection even of the "winning" stimulus, due to a filtering cost (Kahneman, Treisman, \& Burkell, 1983; see Heinke \& Humphreys, 2003, for an explicit simulation).

\section{EXPERIMENT 4 Semantic Effects on Word Targets}

In Experiment 4, the influence of new related distractors was examined again, but in this case, visual similarity effects from the WM stimulus were avoided by using a picture in WM but words in the search display (as in Experiment 2). There were seven display types. On targetabsent trials, there were four conditions: (1) WM-repeat/ same-category (cat + e.g., pig), (2) WM-repeat/different category (cat + e.g., pen), (3) neutral same (e.g., pig + hen), and (4) neutral different (e.g., pen + hat). Hence, on absent trials, semantic similarity between the distractor and the WM cue and the target was present in three conditions $(1,2$, and 3$)$. If there are effects of the WM stimulus over and above such effects, RTs should be lengthened when the WM stimulus reappeared in the search display
(Conditions 1 and 2 above), relative to when it was absent (Condition 3). On target-present trials, there were three display types: (1) neutral same (dog + e.g., pig), (2) neutral different (dog + e.g., pen), and (3) WM repeat (dog + cat). On target-present trials, the effect of a new related distractor would be demonstrated by the difference between trials with a new animate distractor and trials on which the distractor was a new inanimate object (neutral same vs. neutral different).

\section{Method}

The memory stimulus was always a picture of a cat. The search display contained two printed words. The task was to detect the presence of the word $d o g$. When present, the target was paired with the word cat (WM condition), with another animal name ( pig, hen, owl, cow, and fox), or with the name of an inanimate object (pen, box, $h a t, j u g$, and pan). Note that all the words used here were three letters long. The items selected as animate and inanimate distractors were sampled at random.

On absent trials, the WM cue was presented on half of the trials (paired with the name of either an animate object or an inanimate object). The other half of absent trials were made up by presenting two words corresponding to two animate or two inanimate objects. There were 12 trials in each condition, making a total of 84 trials. Twenty-eight participants ( 23 females, 5 males; median age $=$ 19 years). Figure 7 illustrates the sequence of events on a trial.

\section{Results}

Since the conditions could not be balanced across absent and present trials, separate repeated measures ANOVAs were carried out on RTs for each response type. Targetabsent trials were analyzed in a $2 \times 2$ ANOVA with the factors being WM presence (present/absent) and distractor category (same/different). There were reliable main effects of WM presence $\left[F(1,25)=6.82, p<.015, \eta^{2}=\right.$ $.214]$ and distractor category $[F(1,25)=5.87, p<.023$, $\left.\eta^{2}=.19\right]$. There was no reliable interaction $(F<1)$. RTs were lengthened when the WM item was re-presented in the search display, but in a reversal of the results from Experiment 3, RTs were shortened when the new related distractor was present. RTs on target-present trials were assessed in a one-factor ANOVA. There was a reliable effect of distractor category $[F(2,50)=7.5, p<.006]$. Further comparisons across the conditions showed reli-

Time

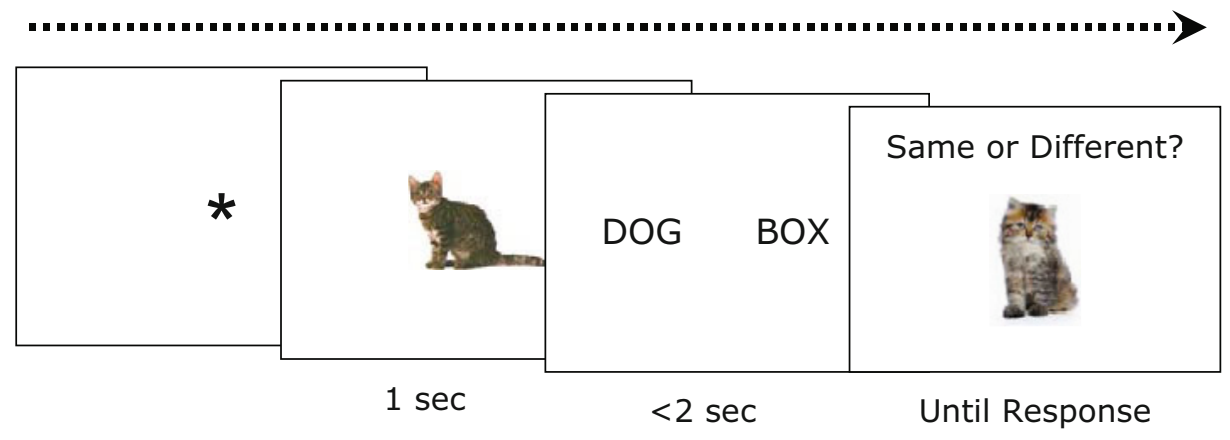

Figure 7. Schematic presentation of a trial in Experiment 4. On this trial, the target (dog) is present along with an inanimate object as a distractor (neutral different condition). A present response is expected. A memory probe is also presented (here, the memory response is different). 


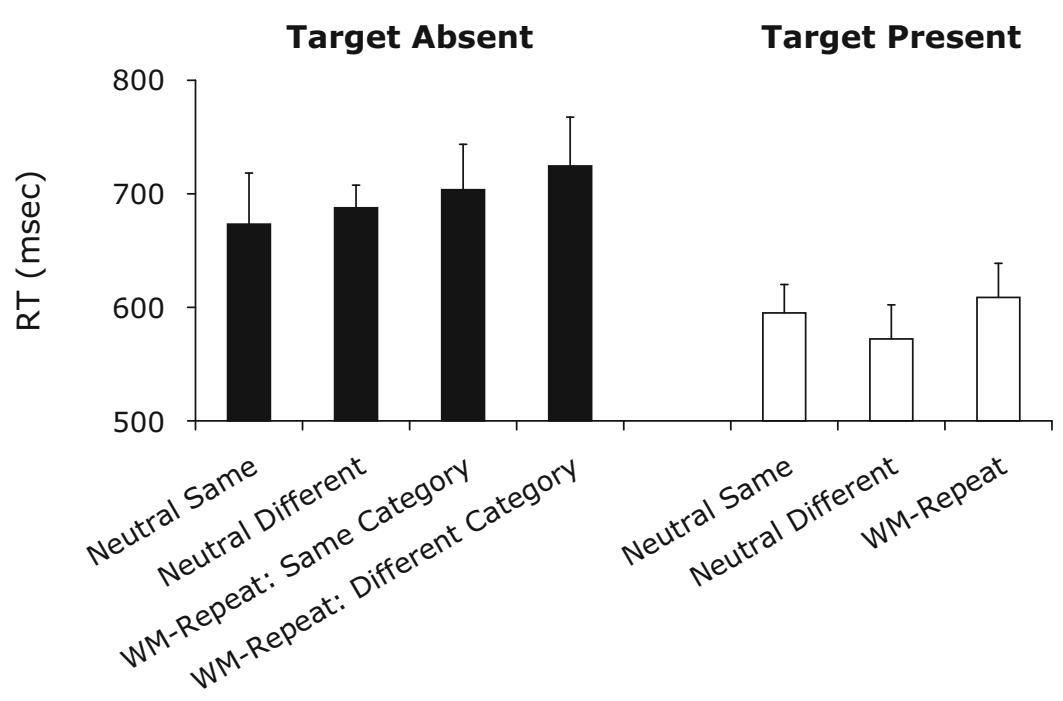

Figure 8. Mean correct response times (RTs, in milliseconds) in Experiment 4 (effect of a new, related distractor with a pictorial working memory [WM] stimulus, and a word search display). Error bars indicate standard errors.

able differences between the average of the WM repeat conditions 5 and, respectively, the neutral same condition $[t(25)=-3.78, p<.01]$ and the neutral different condition $[t(25)=-2.06, p<.001]$. Figure 8 shows the RT data.

The average error rate on search task was less than $1 \%$ for all conditions (Table 1). Accuracy on the memory probe task was $94 \%$, and there was no indication of a speed-accuracy trade-off.

\section{Cross-Experimental Comparison: Effect of the New Related Distractor}

A comparison of the data from Experiments 3 and 4 was carried out to assess both the overall pattern of data and whether this changed as a function of whether the target stimulus was a picture (Experiment 3 ) or a word (Experiment 4). Only target-absent trials were analyzed, because only target-absent trials included an orthogonal manipulation of WM repetition and new distractor relatedness; in addition, effects of the distractors tended to be stronger on absent trials. The overall between-group ANOVA across experiments revealed main effects of WM presence (present/absent) $\left[F(1,52)=55.18, p<.0001, \eta^{2}=.515\right]$ and distractor category (same/different) $[F(1,52)=10.77$, $\left.p<.002, \eta^{2}=.172\right]$. There were two-way interactions between WM presence and experiment $[F(1,52)=36.3$, $\left.p<.0001, \eta^{2}=.411\right]$, between WM presence and distractor category $\left[F(1,52)=5.4, p<.024, \eta^{2}=.095\right]$, and between distractor category and experiment $[F(1,52)=$ $\left.18.57, p<.0001, \eta^{2}=.263\right]$. There was also a tendency for a significant three-way interaction between experiment, WM presence, and distractor category $[F(1,52)=$ $\left.3.5, p<.06, \eta^{2}=.063\right]$. The interaction between WM presence and experiment arose because the effect of repeating the WM stimulus in the search display was greater in Experiment 3 (pictorial target) than in Experiment 4 (verbal target). The interaction between WM presence and distractor category arose because, across the experiments, the effect of the related distractor was weaker when the WM stimulus was repeated (there was an overall interference effect of $35 \mathrm{msec}$ from the related distractor when the WM stimulus was repeated, whereas it was $60 \mathrm{msec}$ when the WM stimulus was absent). The trend to a three-way interaction was due to the new related distractor interfering with the response in Experiment 3, whereas it facilitated performance in Experiment 4 (see Figure 9).

\section{Discussion}

The data indicated again that the presence of the WM stimulus in the search display negatively affected search performance in Experiment 4. The effect was present for both target-absent and target-present trials. In contrast to Experiment 3, the new related distractors speeded performance, as compared with when the new distractors belonged to a different category in Experiment 4. The results with new distractors here contrast also with the effects in Experiment 3 on neutral trials. In contrast to Experiment 3, in Experiment 4, the neutral same condition was slowed, relative to neutral different trials.

Why would new related distractors tend to facilitate performance here when they disrupted search previously? At least two accounts of this result can be supposed. One is that the previous interference effect was caused by the visual similarity of the new related distractors to either the WM or the target. If attention is drawn to items on the basis of whether their visual features match those of stimuli specified in WM (either the irrelevant WM cue here, or the template for the target), visually similar related distractors will compete strongly for selection. On the other hand, semantic distractors might facilitate the processing of other simultaneously presented related items in a display. In the present case, visual similarity effect will be minimized by present- 


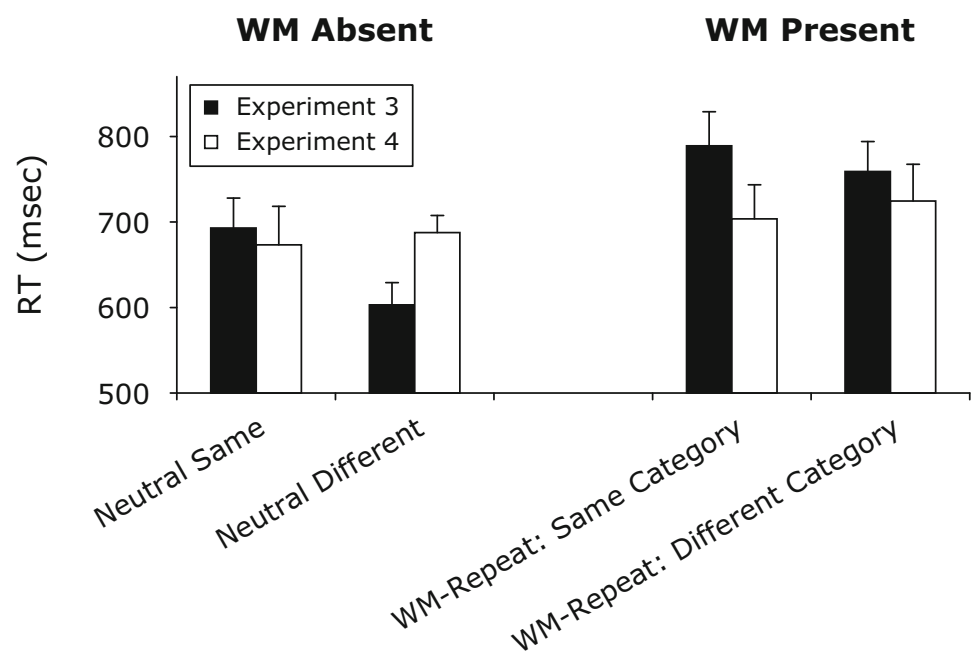

Figure 9. Comparison between mean correct response times (RTs) on targetabsent trials in Experiment 3 (pictorial search display) and RTs on targetabsent trials in Experiment 4 (word search display). Error bars indicate standard errors. WM, working memory.

ing words, and a semantic facilitation effect may then be revealed. However, other researchers who have examined interference effects from related, new distractors have found disruption when the distractors were semantically, but not visually, related to targets (Belke et al., 2008). This suggests that semantic similarity of the targets and distractors can disrupt performance in the same way as the visual similarity.

An alternative proposal is that the difference arises because of differences in the speed of deriving semantic information from pictures (Experiment 3), relative to words (Experiment 4). The new distractor may disrupt performance when semantic information is derived rapidly (from pictures in the search display; Potter \& Faulconer, 1975), since semantic information will be available to attract attention (when it is related to the target and/or the WM cue). With words, semantic information may be derived less rapidly, and so, attention is less likely to be attracted to the new, related distractor. Any small benefits from new, related distractors may then reflect their ease of being rejected as the target, on occasions on which they are selected (i.e., semantic facilitation reflects an effect on distractor rejection, whereas semantic interference arises at a stage of competition for selection with targets). Whichever account is offered for the data with the new, related distractor, the results with the WM item confirm that WM cuing can be based on quite abstract representations of the stimuli and can transfer from picture to words (see also Experiment 2). The contrasting results with the WM and new distractor stimuli also indicate that the effects of the WM stimulus are not due to its acting in the same manner as a new, related distractor (given that, here, the WM item, like the new, related distractor, belonged to the same category as the target). If that was the case, the WM condition should have behaved similarly to the new distractor condition. It did not. We conclude that the two effects are distinct.

\section{EXPERIMENT 5 Effects of Inanimate WM Cue}

In all the experiments reported here, the WM item has come from the same category and has been related to the search target. Although we have argued that the WM effect differs from the new, related distractor effect (Experiment 4), it remains possible that there is some contribution from the WM stimulus being related to the target, rather than from the mere reappearance of the WM stimulus in the search display. To rule out effects of the WM item being related to the target in Experiment 5, the target and the WM stimulus belonged to different categories. In this case, any capture of attention from the WM stimulus cannot be because it is related to the distractor. In addition to this, the new distractor could be related either to the target (as in Experiments 3 and 4) or to the WM stimulus. Would the relationship between the new distractor and the target now be more critical than the relationship between the new distractor and the WM cue?

\section{Method}

The method followed that described in the General Method section, except that the WM cues were randomly drawn from a pool of five different exemplars of cups. There were eight conditions in the experiment, with examples given here for the target dog and the WM item cup. On target-absent trials there were four conditions: (1) neutral/same category as target (pig + hen), (2) neutral/same category as WM (pen + jug), (3) WM-repeat/same category as target (cup + pig), and (4) WM-repeat/same category as WM (cup + jug). On target-present trials, there were four conditions: (1) neutral/ same category as target $(\mathrm{dog}+\mathrm{pig})$, (2) neutral same category as WM (dog + jug), (3) WM-repeat/same exemplar (dog + cup), and (4) WM-repeat/different-exemplar (dog + other cup).

Twenty-eight participants ( 23 females, 5 males; median age $=$ 19 years) were tested. There were 11 trials in each condition, generating 88 trials per block. The conditions were presented randomly. Figure 10 illustrates the sequence of events on a trial. 


\section{Time}

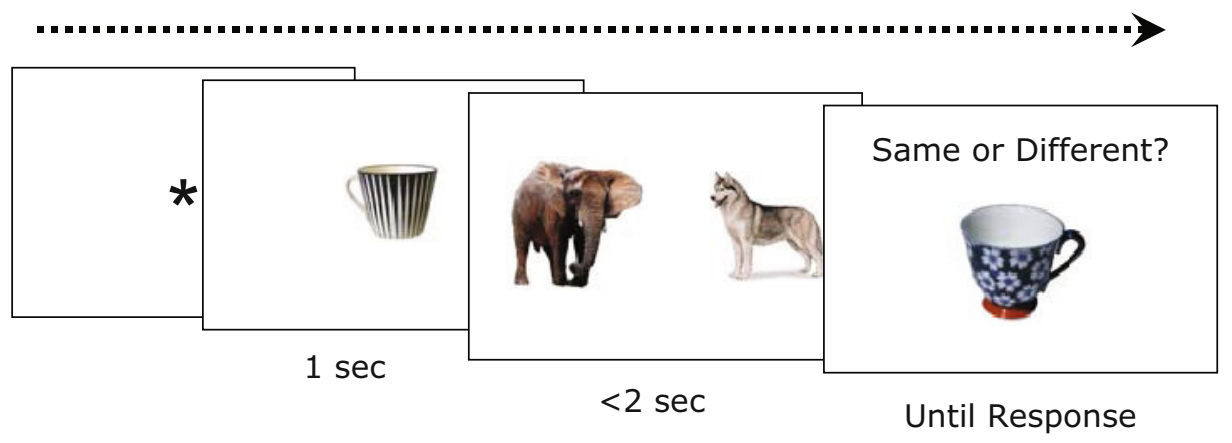

Figure 10. Schematic illustration of a sequence of events making up a trial in Experiment 5. On this trial, a search target (a dog) was presented along with a neutral distractor from the same category (an elephant). The search display is followed by a memory probe (here, the memory response is different).

\section{Results}

Since the conditions were not balanced, the data for absent and present trials were analyzed independently. Absent trials were analyzed in a $2 \times 2$ ANOVA, with the factors being WM cue (present or absent) and distractor category (animate, inanimate). There were reliable main effects of both factors [WM cue, $F(1,27)=31.28, p<$ $.0001, \eta^{2}=.538$; distractor category, $F(1,27)=36.7, p<$ $\left..001, \eta^{2}=.576\right]$. There was no interaction between WM presence and distractor category $(F<1)$. RTs were lengthened when the WM cue reappeared in the search display, and they were longer when the distractor belonged to the same category as the target. These two effects exerted an additive effect on performance (see Figure 11).
For target-present trials, a one-factor ANOVA showed no reliable difference between the four conditions $[F(3,81)=2.19, p>.095]$.

The average error rate on search task was never more than $3.8 \%$ in any condition (see Table 1). Accuracy for the memory probe was high (93\%), showing good encoding of the WM cue. There was no effect of whether the WM item reappeared in the search display $(F<1)$.

\section{Discussion}

These results demonstrated several points. First, the data indicated an effect of the memory cue's reappearing in the search display on trials on which the target was absent, even though the WM item now belonged to a dif-

\section{Target Absent}

\section{Target Present}

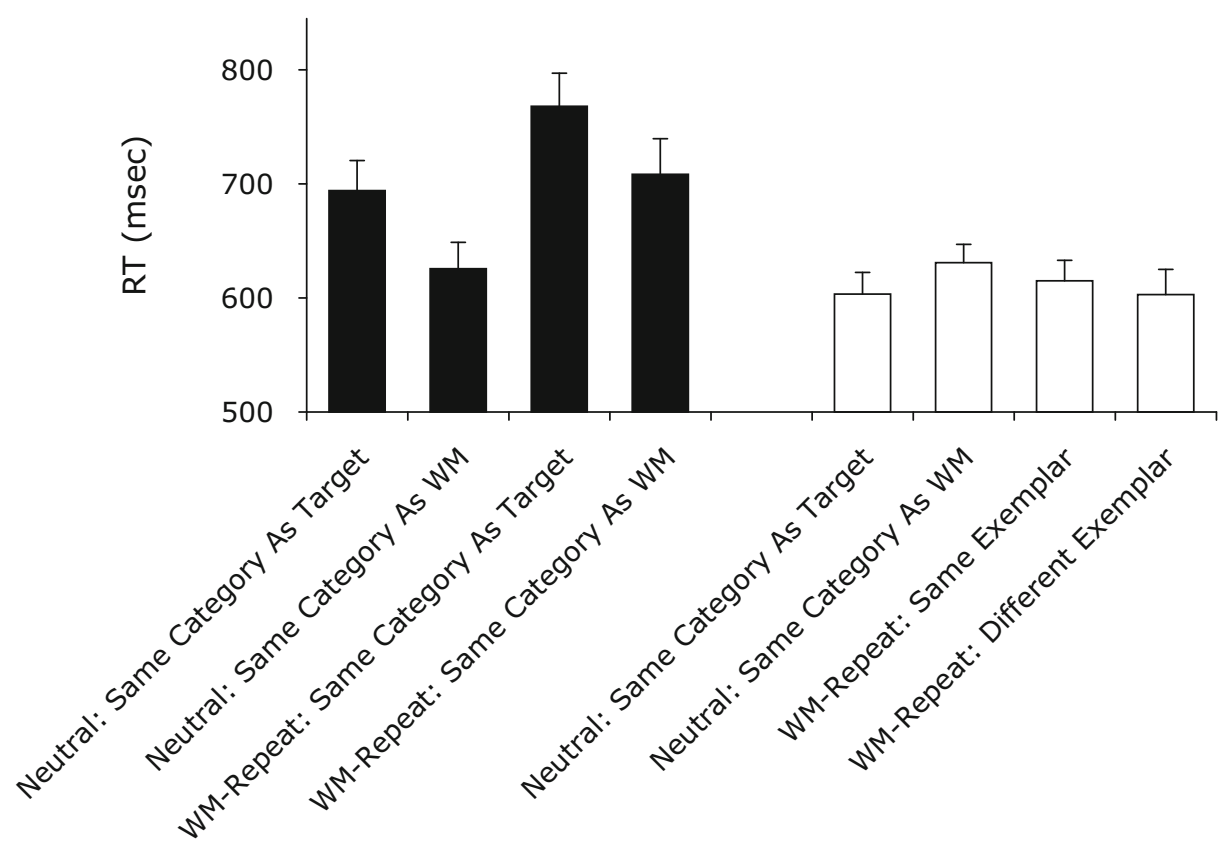

Figure 11. Mean correct response times (RTs, in milliseconds) in Experiment 5, where the working memory (WM) cue and search target differ in category. Error bars indicate standard errors. 
ferent category, relative to the target. RTs were longer when the memory item reappeared, relative to when the stimulus was replaced with another item from the same category (neutral same WM). There was no reliable effect on target-present trials, though. This suggests that when there was a category difference between the memory and target stimuli, the target was sufficiently salient to be selected without strong competition from the WM cue.

In addition to the effect of re-presenting the WM cue on absent trials, there was also an effect of presenting a new distractor that belonged to the same category as the target - the related distractor effect (cf. Moores et al., 2003). RTs were longer in this case than when the new distractor was related to the WM cue (neutral same category as target $>$ neutral same category as WM). Thus, the similarity of the new distractor to the attributes of the expected target was more critical than its similarity to the item held in WM. Note, though, that the target in this study was an animate item, and the effect of presenting a new distractor from the same category may be due to its visual, rather than its semantic, similarity to the target. In contrast to this, the effects of the WM cue here indicate that the memory item does not need to belong to the same category as (or to be visually similar to) the target in order to capture attention when it reappears in search.

On absent trials, the effect of having the distractor that came from the same category as the target combined additively with the effect of re-presenting the WM cue in search display. This is an interesting result that helps to define the relation between the WM effect and the related distractor effect, since it suggests that the two effects reflect independent forms of competition for target selection. The independent enhanced competition (when both types of distractor are present) could lead to filtering costs and slower target-absent trials (cf. Kahneman et al., 1983). Alternatively, the slowed absent responses when both distractors are present may reflect disengagement costs. Here, we suppose that it takes increased time to reject a WM cue or a related distractor, relative to an unrelated distractor (see Soto et al., 2006a). When both types of distractor are present, there will be a disengagement cost on every trial once one distractor is selected. When only one of these distractors is present (and the other distractor is unrelated), the disengagement cost will be reduced on those trials on which the unrelated distractor is selected (even if the distractor is selected on a minority of trials).

\section{EXPERIMENT 6 Associative Relations Between Target and Distractors}

In all the experiments reported to date, the related distractor has always belonged to the same category as the target. The target has also always been an animate object (a dog). Animate pictures tend to be visually similar (see Humphreys, Riddoch, \& Quinlan, 1988). Hence, with pictorial search stimuli, related distractors tended to be visually, as well as semantically, similar to targets. Experiment 6 was designed to assess whether this was important.
In Experiment 6A, we introduced related distractors that were associatively related but from a category different from that of the targets (e.g., target $=$ dog, related distractor $=$ kennel). These associated distractors have minimal physical similarity to targets. Despite this, previous authors have shown that these associative, related distractors disrupt search (Belke et al., 2008; Moores et al., 2003). We assessed whether these associative, related distractors disrupt performance here. To avoid effects of either semantic or visual similarity effects to the target in the WM condition, the WM stimulus belonged to a category different from that of the target and the related distractor (as in Experiment 5).

Experiment 6B used the same stimuli as those in Experiment 6A, except that we swapped the WM and target items. Here, for the first time, the target was an inanimate object (a cup). In this case, however, the related distractor was associated to the WM cue, rather than to the target. This enabled us to test whether the WM cue had a semantic, as well as visual, effect with pictorial stimuli. Effects from pictorial cues in WM on word targets (e.g., Experiments 2 and 4 here), or vice versa (Soto \& Humphreys, 2007), have been taken to indicate effects of semantic information held in WM, but in such cases, there has always been an exact match between the identity of the WM cue and its representation in the search display (e.g., picture of cat, the word cat). Here, we tested for the first time whether there is also some generalization to other semantically related stimuli (related distractors). If that is the case, then, relative to trials on which only unrelated distractors are present, RTs will be lengthened when a related distractor occurs even in the absence of the WM cue.

\section{Method}

Thirty students participated, 15 in Experiment 6A (5 male, 10 female; median age $=22.4$ years $)$ and 15 in Experiment $6 \mathrm{~B}$ ( 7 male, 8 female; median age $=24.1$ years). One participant's data were corrupted in Experiment 6A and, therefore, were excluded from the analysis.

For Experiment 6A, the target was a dog and the WM cue a particular exemplar of a cup. The target-related distractors were a dog basket, a dog collar, a bone, and a kennel. The unrelated distractors were vehicles (a car, a lorry, a tractor, and a motorbike). The conditions were as follows. On target-absent trials, there were four conditions: (1) two related (kennel + bone), two objects that were semantically related and visually dissimilar to the target; (2) two unrelated (car + lorry); (3) WM and related distractor (cup + kennel); and (4) WM and unrelated distractor (cup + car). On target-present trials, there were three conditions in which the target appeared together with one of the following stimuli: (1) a related distractor (dog + kennel), (2) an unrelated distractor (dog + car), or (3) the WM item (dog + cup). Experiment 6B was the same as Experiment $6 \mathrm{~A}$, except that the target was a cup and the WM was a dog. The other distractors matched those in Experiment 6A, with the result that the related distractor was now associated with the WM cue, and not the target.

In both experiments, there were 126 trials per block (18 trials in each condition). The conditions were presented randomly. Each participant carried out one block of trials. Figure 12 illustrates the sequence of events on a trial. On memory probe trials, either the same or a different exemplar of the WM cue was presented. In Experiment $6 \mathrm{~A}$, this was the same or a different cup, whereas in Experiment $6 \mathrm{~B}$, it was the same or a different dog. 


\section{Time}

Experiment $6 \mathrm{~A}$

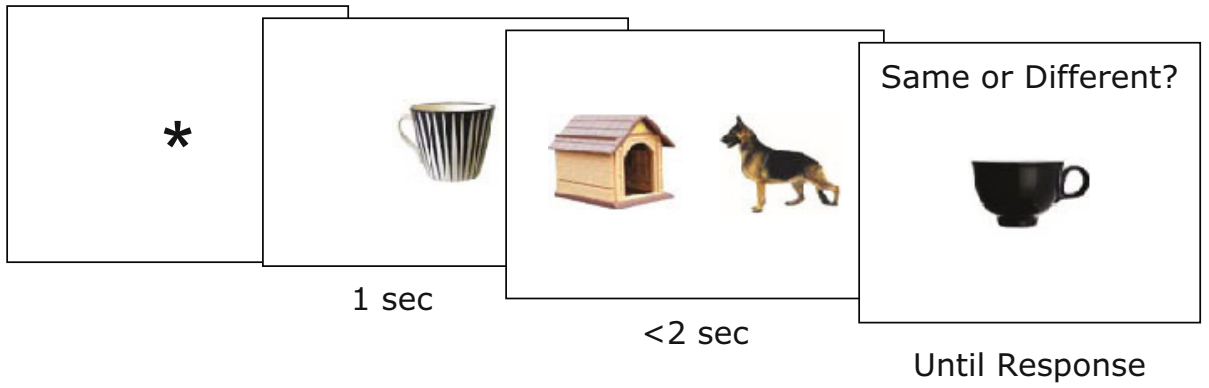

Experiment 6B

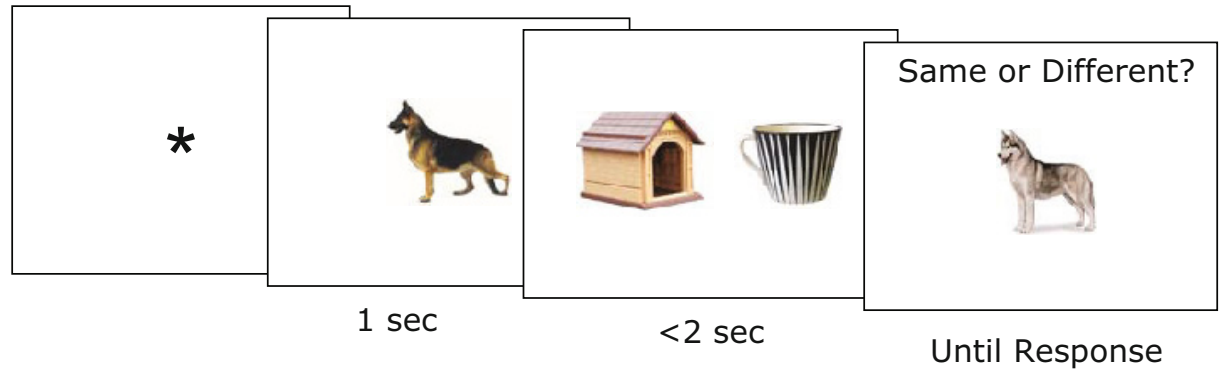

Figure 12. Schematic illustration of a sequence of two typical trials in Experiments 6A and 6B. The upper figure depicts a target-present trial in Experiment 6A, where the target (dog) appears together with an object that is semantically related to it (a kennel). The search display is followed by a memory probe in which a cup different from the working memory (WM) item is presented. In the lower part of the figure, a target-present trial in Experiment 6B is depicted. The target (cup) is now presented with a distractor that is semantically related to the WM cue. The search display is followed by a memory probe, in which a dog different from the WM dog is presented.

\section{Results}

Since the conditions could not be balanced across absent and present trials, separate repeated measures ANOVAs were carried out on RTs for each trial type and for each section of Experiment 6.

RT analysis. Separate repeated measures ANOVAs were conducted on correct RTs from target-absent and target-present trials in Experiments 6A and 6B.

Experiment $6 \mathrm{~A}$. A $2 \times 2$ ANOVA was conducted on target-absent trials, with the factors being WM presence (present/absent) and semantic relation (semantically related/unrelated). There was a significant main effect of WM presence $\left[F(1,14)=22.56, p<.0001, \eta^{2}=.617\right]$; WM-present trials were $147 \mathrm{msec}$ longer than WM-absent trials. There was also a significant main effect of semantic relation $\left[F(1,14)=5.05, p<.042, \eta^{2}=.264\right]$; trials containing a target-related distractor took, on average, $49 \mathrm{msec}$ longer than trials without such a distractor. There was no interaction between WM presence and the related distractor effect $(F<1)$. On target-present trials, a simple three-factor ANOVA showed no significant differences between the trials $(F<1)$.

Experiment $6 B$. As in Experiment 6A, a $2 \times 2$ ANOVA was conducted on RTs from target-absent trials, with the factors being WM presence (present/absent) and semantic relation (semantically related/unrelated). There were again main effects of WM presence $[F(1,15)=22.3, p<$ $\left..0001, \eta^{2}=.604\right]$ and related distractor $[F(1,15)=10.13$, $\left.p<.006, \eta^{2}=.403\right]$. RTs were $194 \mathrm{msec}$ longer when the WM cue was re-presented and $54 \mathrm{msec}$ longer when the related distractor was present. There was also an interaction between WM presence and the related distractor effect $\left[F(1,15)=26.25, p<.0001, \eta^{2}=.636\right.$; see Figure 13] When the WM cue was absent, RTs were longer with related distractors than with unrelated distractors $[t(15)=$ $-7.4, p<.0001]$. When the WM was present, there was no effect of the related distractor $(t>1)$.

A simple three-factor ANOVA on target-present trials failed to show any significant differences between the trials $(F<1)$. Figure 13 shows the RTs in Experiments 6A and $6 \mathrm{~B}$.

The errors are presented in Table 1. The error rate was relatively high when related distractors appeared on target-present trials. This suggests that there was a bias to respond "absent" if a related distractor was selected, both when the distractor was related to the target and when it was related to the WM cue (in Experiments 6A and 6B). There were no other signs of a speed-accuracy trade-off.

\section{Discussion}

Experiment 6 replicated the results in prior studies of the related distractor effect (e.g., Belke et al., 2008; 
Target Absent

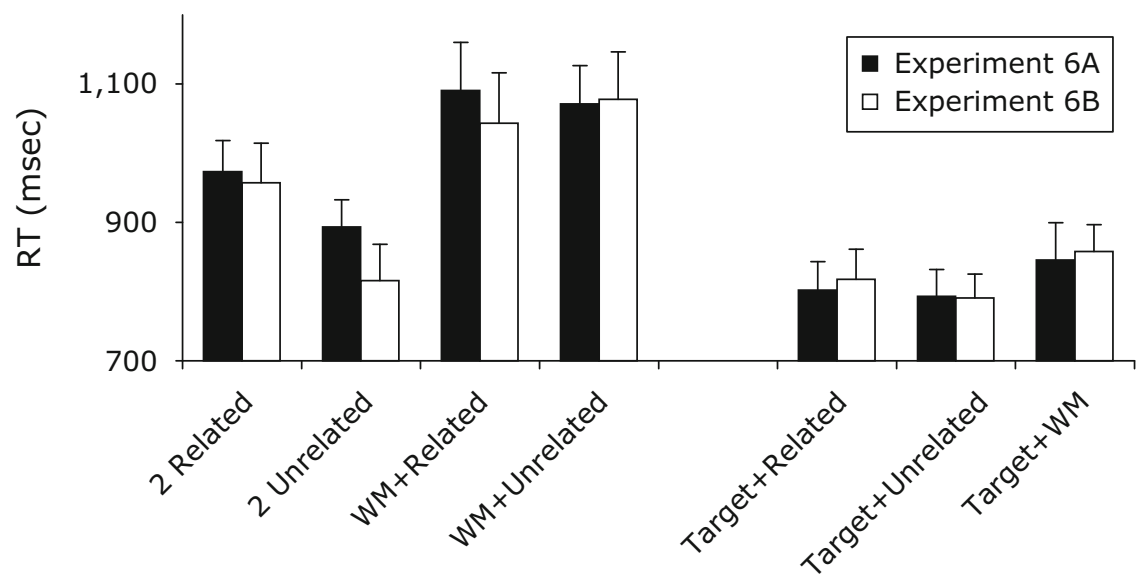

Figure 13. Mean correct response times (RTs, in milliseconds) from target-absent and target-present trials in Experiments 6A and 6B. Error bars indicate standard errors. WM, working memory.

Moores et al., 2003) in demonstrating that search can be disrupted by the presence of a related distractor in the display (even when the effects of physical similarity are minimized). In Experiment 6A, the critical distractor was related to the search target. In this case, there were additive effects of the presence of the related distractor and the WM cue (on target-absent trials). This matches the data from Experiment 5 and again suggests that there are independent sources of competition for selection-from items held in WM and from items that are associatively related to search targets.

In Experiment 6B, the related distractor was now associated with the WM item, and the target was unrelated to both. In this case, the related distractor still disrupted performance when the WM cue was absent. This confirms that semantic information held in WM can influence search (Experiments 2 and 4 here; Soto \& Humphreys, 2007). However, there was no effect of the related distractor when the WM cue appeared; in this case, there was only a disruptive effect of the WM cue. This indicates that a distractor that is semantically related to the WM cue competes less with that cue (Experiment 6B) than when the distractor is semantically related to the target (Experiment 6A), when it forms an independent source of competition to the WM cue.

\section{GENERAL DISCUSSION}

We have reported six experiments examining the effects of an item held in WM on selection of a subsequent target. Experiments 1 and 2 examined performance when all the stimuli belonged to the same category. Experiment 1 showed that a pictorial exemplar held in WM disrupted search for a related target when the exemplar reappeared in the search display. This disruption was over and above that found when another item from the same category reappeared in the search display (the neutral condition).
Interestingly, the disruptive effect of the WM stimulus was just as strong when a different exemplar of this item reappeared in the search display as when the same exemplar reappeared, even though memory was tested for the specific exemplar that had been shown. These data indicate that guidance from WM can be based on less specific information than that held in WM. Experiment 1 also included a baseline condition when participants passively viewed the first stimulus (Experiment 1A). Under this circumstance, the effect of the WM item was reduced (see also Soto et al., 2005). This provides evidence for the effects' being enhanced from a representation of the cue held in WM.

Experiment 2 assessed performance when the item in WM and the search stimulus were presented in different modalities (WM picture, search on words). The effects of the WM item were again apparent. This cross-modal effect cannot be caused by bottom-up priming from the cue or by physical similarity between the cue and the target. Instead, the data suggest that participants hold semantic, as well as visual, properties of stimuli in WM and that these semantic features may be activated by the items in the search display, with the result that attention is biased to stimuli with matching features (see also Soto \& Humphreys, 2007).

Experiments 3-6 examined effects on search, not only of repeating an item held in WM in a search display, but also of presenting new, related distractors (related to either the target [Experiments 3-5 and 6A] or the WM cue [Experiment 6B]). Experiment 6A showed that this interfering effect was not due just to visual similarity to the target, since the disruptive effect occurred with visually unrelated, associative distractors too. Experiment 6B also revealed effects of whether the distractor was related to the WM stimulus as well as to the target. This again provides support for matching's being based on irrelevant information in WM, as well as on relevant target attributes, and for this effect operating at a semantic level. 


\section{The Relations Between WM-} and Target-Related Interference

There was an additive combination of having a distractor in the search display that matched with a WM cue and having a distractor present in the display that was related to the target. We can think of the combined cost on these trials in one of two ways. On one account, there is a filtering cost (Kahneman et al., 1983), due to both distractors serving as strong competitors for selection. When two competing distractors are present, there may be a sharing of attentional weights between them (cf. Duncan \& Humphreys, 1989), retarding the selection of both items and slowing absent responses in particular. Bundesen's (1990) theory of visual attention (TVA) provides a formal account of this. In TVA, stimuli attract attentional weight if they match a template in WM. When two stimuli provide an equal match (one related to the target representation in WM, the other to the irrelevant WM stimulus), they will attract equal weights that are less than when only one such distractor is present, and hence, the selection of either stimulus will be delayed and interference linked to these specific distractors will be reduced. Any effects of the distractors will still be greater on absent than on present trials, because the distractors do not have to compete against the target on absent trials. On present trials, the target will typically win the competition for selection, since it has the highest search-specific attentional weight. The alternative account attributes the slowing to delayed disengagement of attention from a distractor that is related either to the cue in WM or to the target. This account assumes that absent responses are made after selecting one or both distractors. If absent responses follow the selection of both distractors, RTs will be lengthened by each critical distractor, when both are present. If absent responses follow the selection of a single item, RTs will still be delayed, since one of the two distractors selected will be related to the cue or the target, when both critical distractors are present. When one distractor is unrelated, absent trials will be delayed less on those (even minority) trials when this item is selected. In either case, the results are consistent with there being independent bias on selection, from $\mathrm{WM}$ and from semantic associates of targets.

The one exception to the costs from related distractor occurred in Experiment 4, when word targets were presented. In this instance, the presence of a distractor related to the target facilitated RTs on neutral different trials. This positive influence of the related distractor again distinguished the related distractor effect from the effect of repeating an irrelevant cue held in WM. Given that, with pictorial targets, associated distractors slowed performance (Experiment 6), this result can be attributed to the use of word targets in Experiment 4. We suggest that, with word displays, a semantic distractor does not compete strongly for selection, perhaps because the words are represented phonologically, rather than semantically (Coltheart et al., 2001). Any speeding of response, when the related distractor is present, may reflect its easier re- jection when it is semantically related to but phonologically different from the target.

\section{Generalization Across Exemplars}

One other result to note is that the effects of attentional guidance from the item in WM generalized across exemplars (indeed, in Experiment 1, effects on present trials were significant only when the WM stimulus reappeared as a different exemplar). On the other hand, memory was probed for the specific exemplar presented on the trial, and memory performance was good. This indicates that the process of guiding attention from WM seems not to be tied to the detailed properties of the stimulus in WM, even though these properties are being maintained in WM. In some sense, attentional guidance is coarser than the representation held in WM itself. The data on cross-modal and semantic guidance (Experiment 2 and Experiment 6B, respectively) also highlight that guidance is based on relatively abstract representations of stimuli in WM. Recent accounts of WM stress that it maintains not only specific visual and phonological representations of stimuli, but also "episodic" codes that integrate across representations in modality-specific "slave systems" (Baddeley, 2000). Our results are consistent with attentional guidance operating from these more abstract episodic codes, and not from the "slave" representations themselves.

Irrespective of this, the generalization of the WM effects across exemplars provides strong evidence against guidance being a voluntary process (e.g., stemming from participants wishing to "refresh" their representation of the item held in WM). When the repeated item was a different exemplar from the original cue, attention should be biased against the repeated stimulus, since this would disrupt memory for the specific exemplar. Despite this, the effects on attentional guidance were as strong with different as with the same exemplars. This means that if reappearance of the WM item in the search has been used as a deliberate strategy for updating the memory, it has not been the cause of the effect of WM item on search here. Note also that, in the procedure used here, the cue was always invalid (always falling at a different location from the target), yet a robust cuing effect occurred. This strongly suggests that the effects are involuntary (see also Soto et al., 2005). The effects of reappearance of the WM item in the search display also arose when the WM stimulus belonged to a different category from the target (in Experiments 5 and $6 \mathrm{~B}$, when the target was animate and the WM stimulus inanimate), so the result is not dependent on the properties of the WM stimulus overlapping with the set for the target. Furthermore, the evidence for an additive relation between the WM and related distractor effects suggest that there is independent guidance from an irrelevant item in WM and from a target. This would fit with participants compartmentalizing "relevant" (targetrelated) and "irrelevant" (WM cue) parts of WM, even if under current conditions, they cannot prevent some guidance from the irrelevant WM stimulus. 


\section{Guidance of Search}

Although we did not manipulate the display size in this study, this has been done in prior works on distractor interference effects. Belke et al. (2008) studied the effect of having a distractor associatively related to the target and showed that distractor interference was additive across display sizes of four and eight items. This indicates that semantic information can be derived across displays of up to eight items, to then compete for selection with the target. Soto et al. (2005) varied the display size when examining the effects of an irrelevant item in WM. Display size effects on search were reduced when the target was validly cued by the reappearance of the WM distractor.

In the present study, effects of top-down guidance seemed to occur in parallel across the display, to facilitate search when guidance was valid. The data suggest parallel effects of distractor (in the search display and in WM) across search displays with varying numbers of items.

Finally, although the evidence we have presented has indicated that relatively abstract representations of stimuli can guide search, we do not mean to say that all guidance is abstract in nature; for example, there is clear evidence for visual guidance from target representations (e.g., Vickery, King, \& Jiang, 2005; Wolfe, Horowitz, Kenner, Hyle, \& Vasan, 2004). We suggest that both visual and more abstract representations of stimuli can guide search, consistent with a view that target search templates operate through a multimodal WM system (Baddeley, 2000).

\section{AUTHOR NOTE}

This work was supported by a grant from the ESRC to the third author. Correspondence concerning this article should be addressed to A. B. Balani, Behavioural Brain Sciences Centre, School of Psychology, University of Birmingham, Birmingham B15 2TT, England (e-mail: a.b.balani@bham.ac.uk).

\section{REFERENCES}

BAdDELEy, A. D. (2000). The episodic buffer: A new component of working memory? Trends in Cognitive Sciences, 4, 417-423.

Belke, E., Humphreys, G. W., Watson, D. G., Meyer, A. S., \& TellING, A. (2008). Top-down effects of semantic knowledge in visual search are modulated by cognitive but not perceptual load. Perception \& Psychophysics, 70, 1444-1458.

Boucart, M., \& Humphreys, G. W. (1994). Attention to orientation, size, luminance, and color: Attentional failure within the form domain. Journal of Experimental Psychology: Human Perception \& Performance, 20, 61-80.

Bundesen, C. (1990). A theory of visual attention. Psychological Review, 97, 523-547.

Chelazzi, L., Miller, E. K., Duncan, J., \& Desimone, R. (1993). A neural basis for visual search in inferior temporal cortex. Nature, 363, 345-347.

Collins, A. M., \& Quillian, M. R. (1969). Retrieval time from semantic memory. Journal of Verbal Learning \& Verbal Behavior, 8 , 240-248.

Coltheart, M., Rastle, K., Perry, C., Langdon, R., \& Ziegler, J. (2001). The DRC model: A model of visual word recognition and reading aloud. Psychological Review, 108, 204-258.

DownING, P. E. (2000). Interactions between visual working memory and selective attention. Psychological Science, 11, 467-473.

Downing, P. E., \& DodDs, C. M. (2004). Competition in visual working memory for control of search. Visual Cognition, 11, 689-703.
Duncan, J., \& Humphreys, G. W. (1989). Visual search and stimulus similarity. Psychological Review, 96, 433-458.

DunCAN, J., HumphreYs, G. W., \& WARD, R. (1997). Competitive brain activity in visual attention. Current Opinion in Neurobiology, 7, 255261

HeinKe, D., \& Humphreys, G. W. (2003). Attention, spatial representation and visual neglect: Simulating emergent attention and spatial memory in the selective attention for identification model (SAIM). Psychological Review, 110, 29-87.

Hodsoll, J., \& Humphreys, G. W. (2001). Driving attention with the top down: The relative contribution of target templates to the linear separability effect in the size dimension. Perception \& Psychophysics, 63, 918-926.

Hodsoll, J., \& Humphreys, G. W. (2005). Preview search and contextual cuing. Journal of Experimental Psychology: Human Perception \& Performance, 31, 1346-1358.

HoutKamp, R., \& RoElfsema, P. R. (2006). The effect of items in working memory on the deployment of attention and the eyes during visual search. Journal of Experimental Psychology: Human Perception \& Performance, 32, 423-442.

HuANG, L., \& PAshler, H. (2007). Working memory and the guidance of visual attention: Consonance-driven orienting. Psychonomic Bulletin \& Review, 14, 148-153.

Humphreys, G. W., Riddoch, M. J., \& Quinlan, P. T. (1988). Cascade processes in picture identification. Cognitive Neuropsychology, $\mathbf{5}, 67-103$.

Kahneman, D., Treisman, A., \& Burkell, J. (1983). The cost of visual filtering. Journal of Experimental Psychology: Human Perception \& Performance, 9, 510-522.

Moores, E., Laiti, L., \& Chelazzi, L. (2003). Associative knowledge controls deployment of visual selective attention. Nature Neuroscience, 6, 182-189.

Moores, E., \& MaXwell, J. (2008). The role of prior exposure in the capture of attention by items in working memory. Visual Cognition, 16, 675-695.

OLIVERS, C. N. L. (2009). What drives memory-driven attentional capture? Journal of Experimental Psychology: Human Perception \& Performance, 35, 1275-1291.

Olivers, C. N. L., Meijer, F., \& Theeuwes, J. (2006). Feature-based memory-driven attentional capture: Visual working memory content affects visual attention. Journal of Experimental Psychology: Human Perception \& Performance, 32, 1243-1265.

Potter, M. C., \& Faulconer, B. A. (1975). Time to understand pictures and words. Nature, 253, 437-438.

RidDoch, M. J., \& HumphreYs, G. W. (1987). A case of integrative visual agnosia. Brain, 110, 1431-1462.

Soto, D., Heinke, D., Humphreys, G. W., \& Blanco, M. J. (2005). Early, involuntary top-down guidance of attention from working memory. Journal of Experimental Psychology: Human Perception \& Performance, 31, 248-261.

Soto, D., \& Humphreys, G. W. (2006). Seeing the content of the mind: Enhanced awareness through working memory in patients with visual extinction. Proceedings of the National Academy of Sciences, 103, 4789-4792.

Soto, D., \& Humphreys, G. W. (2007). Automatic guidance of visual attention from verbal working memory. Journal of Experimental Psychology: Human Perception \& Performance, 33, 730-757.

Soto, D., \& Humphreys, G. W. (2008). Stressing the mind: The effect of verbal suppression and cognitive load on attentional guidance from working memory. Perception \& Psychophysics, 70, 924-934.

Soto, D., Humphreys, G. W., \& Heinke, D. (2006a). Dividing the mind: The necessary role of the frontal lobes in separating memory from search. Neuropsychologia, 44, 1282-1289.

Soto, D., Humphreys, G. W., \& HeinKe, D. (2006b). Working memory can guide pop-out search. Vision Research, 46, 1010-1018.

VICKERY, T. J., KING, L. W., \& JIANG, Y. H. (2005). Setting up the target template in visual search. Journal of Vision, 5, 81-92.

Wolfe, J. M., Horowitz, T. S., Kenner, N., Hyle, M., \& Vasan, N. (2004). How fast can you change your mind? The speed of top-down guidance in visual search. Vision Research, 44, 1411-1426.

Woodman, G. F., LucK, S. J., \& Schall, J. D. (2007). The role of work- 
ing memory representations in the control of attention. Cerebral Cortex, 17, 118-124.

\section{NOTES}

1. Olivers (2009) has recently reported conditions in which the effect of the WM is reduced, which include when the search target changes on every trial. Under these conditions, capacity appears to be taken away from the memory stimulus, making it less effective in search (see also Downing \& Dodds, 2004; Soto \& Humphreys, 2008; Woodman, Luck, \& Schall, 2007).

2. We use the term search display here primarily to distinguish the search display (that participants respond to) from the initial display. However, in all the experiments, we presented only two items, so the experiments do not address whether any effects change search efficiency. We elaborate on the relations between distractor interference in search in the General Discussion section.
3. There is a large body of work showing that there is automatic identification of a single object presented at fixation (e.g., Boucart \& Humphreys, 1994), so automatic identification is to be expected even in a passive view condition.

4. If anything, the effects with a different exemplar were larger than those with the same exemplar. It is not clear why this should be, but the data indicate that the effects were certainly not smaller with a different exemplar.

5. The RT data from both the WM-repeat/same-category and WM-repeat/different-category conditions were averaged and used in the $t$ test calculation against neutral condition, because the two WM conditions did not differ $(t<1)$

(Manuscript received October 13, 2008; revision accepted for publication May 9, 2010.) 